\title{
Aromatase inhibitors in the treatment of oligozoospermic or azoospermic men: a systematic review of randomized controlled trials
}

Mariana A. Ribeiro ${ }^{1}$, Luís F. O. Gameiro², Wellerson R. Scarano ${ }^{1}$, Christine Briton-Jones ${ }^{3}$, Anil Kapoor ${ }^{4,5}$, Mauro B. Rosa6, Regina El Dib ${ }^{5,7}$

\author{
${ }^{1}$ Department of Morphology, Institute of Biosciences, Sao Paulo State University, UNESP, Botucatu, SP, Brazil \\ ${ }^{2}$ Physiotherapy Service School of Medicine, Sao Paulo State University, UNESP, Botucatu, SP, Brazil \\ ${ }^{3}$ Reproductive Medicine Partners of New York, New York, NY, USA \\ ${ }^{4}$ Genito-Urinary Oncology Program, Juravinski Cancer Centre, Hamilton, ON, Canada \\ ${ }^{5}$ McMaster Institute of Urology, at St. Joseph's Healthcare, Hamilton, ON, Canada \\ ${ }^{6}$ Hospital do Coração (HCOR), Sao Paulo,SP, Brazil \\ 'Department of Anesthesiology, Faculdade de Medicina de Botucatu (FMB), Sao Paulo State University, UNESP, \\ Botucatu, SP, Brazil
}

\begin{abstract}
The aim of this study as to analyze published evidence regarding the effectiveness of aromatase inhibitor therapy on improving spermatogenesis in infertile men. We carried out a systematic review of randomized controlled trials. The date of the most recent search was October 4, 2015. Two authors independently selected relevant clinical trials, assessing their methodological quality and extracting data. Three studies were included in this review with a total of 100 participants; however, we were able to include data from only 54 participants in the analysis. In the representation of meta-analysis with a single study comparing testolactone versus placebo, related to the hormone concentrations, there was a statistically significance difference favoring the use of testolactone for Luteinizing Hormone (LH); Estrogen (E2); free Testosterone (free T); free Estrogen (free E2); 17-Hydroxyprogesterone (17OHP); prolactin $(\mathrm{PRL})$. In another analysis from a single study comparing letrozole versus anastrozole, there was also a statistically significance difference favoring the use of letrozole for the increase in both the sperm count and LH. There is only low quality evidence regarding the effectiveness of aromatase inhibitor therapy in infertile men. Further trials are needed with standardized interventions and outcomes.
\end{abstract}

Keywords: aromatase inhibitors, spermatogenesis, infertile men, meta-analysis

\section{INTRODUCTION}

Spermatogenesis is regulated by the interaction of endocrine and paracrine signals, it is dependent on maintenance of high levels of intratesticular testosterone as well as Sertoli cell stimulation with FSH (Jarow \& Zirkin, 2005). Furthermore, LH released by the anterior pituitary binds to receptors on Leydig cells surface and stimulates $T$ production, a steroid hormone which diffuses in the seminiferous tubules (Walker \& Cheng, 2005).

For men with idiopathic infertility, there are no reliable treatments to enhance fertility. However, increased sperm production or motility has been associated with empiric medical therapy using estrogen receptor modulators such as clomiphene citrate or tamoxifen citrate. Such medical therapy to improve spermatogenesis has primarily focused on enhancement of intratesticular testosterone levels and stimulation of FSH production. Unfortunately, use of estrogen receptor modulators results in increased estrogen levels as well as increased testosterone production (Schlegel, 2012).

Aromatase inhibitors (AI) have the ability to increase endogenous testosterone production without the associated increase in circulating estrogens seen with estrogen receptor modulators (Pavlovich et al., 2001). AIs are classified as either steroidal or nonsteroidal. Steroidal inhibitors (such as testolactone, formestane, and exemestane) competitively inhibit aromatase by mimicking androstenedione, causing irreversible enzyme inhibition. Letrozole and anastrozole are nonsteroidal inhibitors that cause reversible enzyme inhibition. Although anastrozole or letrozole suppression is close to $100 \%$ in women, men do not show such a profound decrease, this is probably related to their high plasma $T$ levels (de Ronde \& de Jong, 2011; Stephens \& Polotsky, 2013). Nonetheless, letrozole is a more potent AI than anastrozole (Schlegel, 2012) with both commonly used off-label for treating oligospermia and azoospermia (Stephens \& Polotsky, 2013).

Recent studies have identified a potential specific endocrine defect in men with severe male factor infertility (Pavlovich et al., 2001). Some men with severely impaired sperm production have a relative excess of estrogen to testosterone, quantitatively measured as an increased testosterone/estradiol (T/E) ratio. Pavlovich et al. (2001) characterized men with severe male infertility as having a T/E ratio of 6.9 , whereas men with normal spermatogenesis had a mean T/E ratio of 14.5. Based on these observations, they proposed a cutoff point of 10 as the lower limit of normal T/E ratios in men (calculated using $T$ in $\mathrm{ng} / \mathrm{dL}$, and estradiol as $\mathrm{pg} / \mathrm{mL}$ ). Clinical studies of aromatase inhibitors have focused on men with defective spermatogenesis associated with low serum testosterone levels and abnormal T/E ratios (Schlegel, 2012).

Since males have testosterone levels detected by the pituitary primarily by estrogen levels rather than testosterone alone, inhibition of estrogen production by an aromatase inhibitor can be a potent stimulant for increased $\mathrm{LH}$ production and hence intratesticular and circulating testosterone levels (Santen, 1981). For men with a low serum testosterone and low T/E ratio, treatment with an aromatase inhibitor to increase sperm production would be more rational than treatment with a selective estrogen receptor modulator.

To the best of our knowledge, there is no systematic review comparing the use of aromatase inhibitors on men with impaired spermatogenesis. Therefore, the objective of our study was to review the literature on the effectiveness and safety of aromatase inhibitors in infertile men spermatogenesis. 


\section{MATERIALS AND METHODS}

This systematic review of the literature on intervention studies was carried out in accordance with the PRISMA (Preferred Reposting Items for Systematic Reviews and Meta-analysis) statement (Moher et al., 2009).

\section{Eligibility criteria}

We took into consideration all randomized and quasi-randomized controlled clinical trials evaluating the effectiveness of aromatase inhibitors in the spermatogenesis of infertile men.

The main outcomes measured were sperm count and hormone concentrations (e.g., total estradiol and testosterone levels). Studies were excluded from the review if they were duplicate publications on a study that had already been included, animal studies, case reports or review papers.

\section{Search strategy}

There was no restriction on language, year of publication or publication status. The search was performed in the following electronic databases: the Cochrane database of clinical trials (CENTRAL, the Cochrane Library 2015, issue 5), PubMed (1966-2015), Embase (1980-2015), Lilacs (1982-2015) and Scientific Electronic Library Online (SciELO). The databases were searched for available published and unpublished studies up to October 4, 2015. The search was conducted using multiple combinations of the following key words "aromatase inhibitor", "azoospermia" as well as oligozoospermia" (Table 1).

Table 1. Search strategy

(Azoospermia OR azoospermic OR azoospermic man OR azoospermic men OR Male Infertility OR Male Sterility OR Male Subfertility OR Male Sub-Fertility OR Male Sub fertility OR Oligozoospermia OR Low Sperm Count OR Low Sperm Counts OR Hypospermatogenesis OR Teratozoospermia) AND (aromatase inhibitor OR aromatase inhibitors OR letrozole OR 4,4'-(1H-1,2,4-triazol-1-ylmethylene)- bis(benzonitrile) OR Femara OR Novartis Brand of Letrozole OR Femara OR CGS20267 OR CGS20267 OR Clomiphene OR Clomiphene citrate OR Clomifene OR Clomifen OR Chloramiphene OR Clomid OR Clomide OR Clomiphene Citrate OR Clomiphene Hydrochloride OR Gravosan OR Klostilbegit OR Clostilbegit OR Serophene OR Androxal OR Repros Therapeutic Brand Enclomiphene Citrate OR Dyneric OR Indux OR Aromatase Inhibitors)

\section{Study selection and data extraction}

The titles and abstracts were reviewed by two researchers (MAR and RED) to identify potentially relevant papers. The papers were obtained and independently read in full by the two reviewers. Differences were resolved by discussion and a third party (WRS), if necessary. The main reasons for exclusion were case series and cross-sectional studies. The data was also extracted independently by MAR and RED based on the inclusion and exclusion criteria defined above.

\section{Risk of bias in individual studies}

A risk of bias table, which is a Cochrane measurement tool used to assess the methodological quality of clinical trials, was used as a guide to conduct this systematic literature review (Higgins et al., 2011). We used the following six separate criteria: random sequence generation; allocation concealment; blinding; incomplete outcome data; selective reporting; and other sources of bias.
Summary measurements and synthesis of results

For dichotomous data, we used relative risk (RR) as the effect measurement, with $95 \%$ confidence intervals (CI), along with a fixed-effects model. The null hypothesis of homogeneity across individual studies was tested using the chi-square test and the 12 value.

\section{RESULTS}

The electronic search yielded a total of 2,971 references through database searches. After screening by title and then by abstract, 2,921 papers were excluded due to failure of randomization or lack of appropriate controls, 50 studies were identified as potentially eligible for inclusion in the review. Crosschecking of the references and manual searches did not yield any additional studies for inclusion. Of these, three (Clark \& Sherins, 1989; Gregoriou et al., 2012; Cavallini et al., 2013) studies met the inclusion criteria. Therefore, 42 further references were excluded from this review, as they were either case series or cross-sectional studies (Figure 1 ).

Figure 1. Flowchart of the systematic review

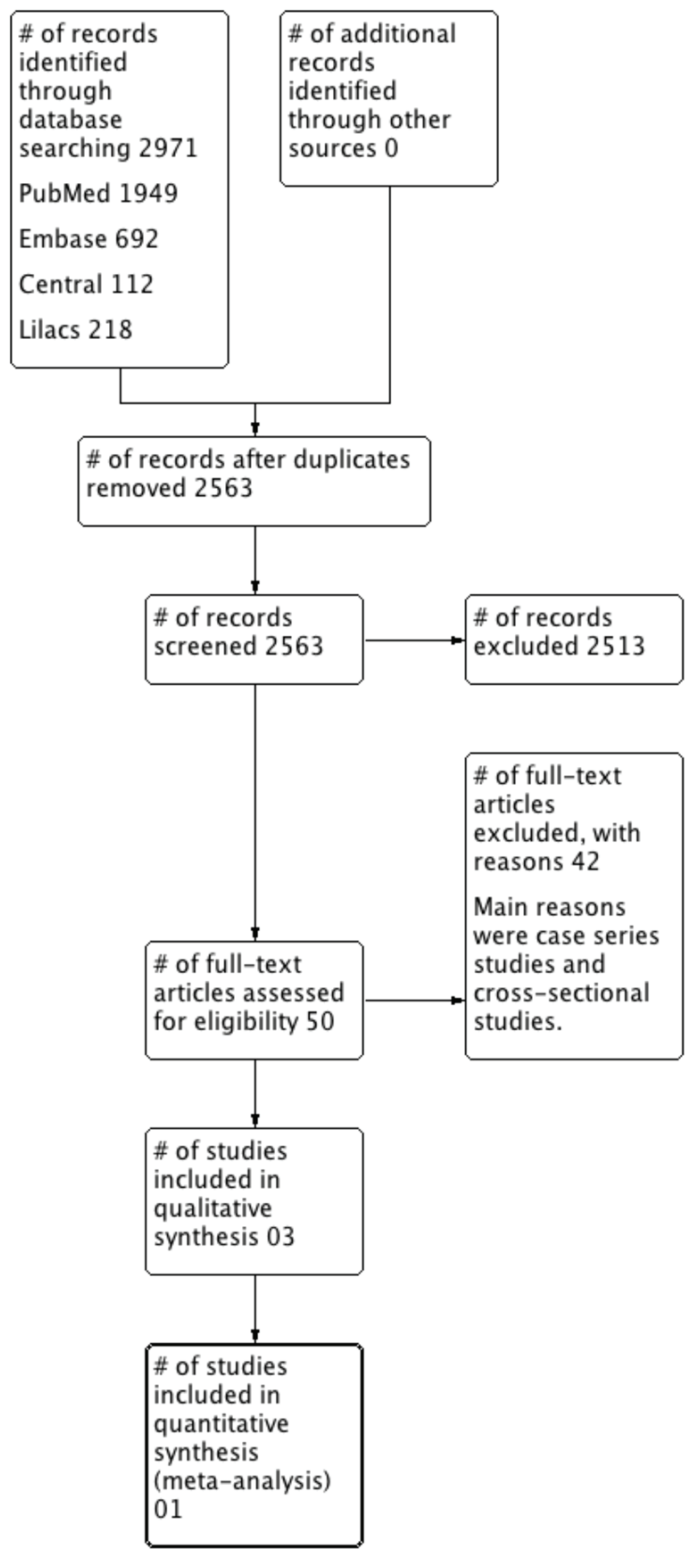




\begin{tabular}{|c|c|c|c|}
\hline & \multicolumn{3}{|c|}{ Author, year } \\
\hline & Clark \& Sherins, 1989 & Gregoriou et al., 2012 & Cavallini, 2013 \\
\hline Location & Atlanta, USA & Athens, Greece & Andros, Italy \\
\hline No. participants & 25 & 29 & 46 \\
\hline $\begin{array}{l}\text { Inclusion } \\
\text { criteria }\end{array}$ & $\begin{array}{l}\text { Men that were partners in infer- } \\
\text { tile marriages in which there was } \\
\text { failure to conceive for at least } \\
\text { two years prior to consideration } \\
\text { of entry into the protocol }\end{array}$ & Men with low T/E2 ratio $(<10)$ & $\begin{array}{l}\text { Non-smoker non- obstructive } \\
\text { azoospermic (NOA) patients who } \\
\text { yielded no spermatozoa with fine } \\
\text { needle and cryptozoospermic pa- } \\
\text { tients with T/E2 ratio < } 10\end{array}$ \\
\hline $\begin{array}{l}\text { Exclusion } \\
\text { criteria }\end{array}$ & $\begin{array}{l}\text { Men with known exposure to } \\
\text { testicular toxins, including che- } \\
\text { motherapeutic agents, radiation, } \\
\text { industrial chemicals, pesticides } \\
\text { and excessive alcohol intake, and } \\
\text { patients with a clinically appar- } \\
\text { ent varicocele, history of crypt- } \\
\text { orchidism or mumps orchitis, } \\
\text { evidence of hypogonadotropic } \\
\text { hypogonadism, or a demonstra- } \\
\text { ble chromosomal abnormality. }\end{array}$ & Not reported & $\begin{array}{l}\text { Seminal white blood cell concen- } \\
\text { tration greater than } 106 \text { ml and/or } \\
\text { a positive seminal cultural analysis } \\
\text { or positive urethral swab chlamyd- } \\
\text { ia test; drug tobacco, or alcohol } \\
\text { abuse; on going medical treat- } \\
\text { ment (gonadotropins, anabolic } \\
\text { steroids, cancer chemotherapy, } \\
\text { non steroidal anti inflammatory } \\
\text { drugs; previous cancer radiother- } \\
\text { apy or chemotherapy, palpable } \\
\text { varicocele; X-ray exposure in the } \\
\text { previous } 8 \text { months; Y chromosome } \\
\text { microdeletion, and karyotype al- } \\
\text { terations if Klinefelter syndrome }\end{array}$ \\
\hline $\begin{array}{l}\text { Type } \\
\text { intervention }\end{array}$ & $\begin{array}{l}\text { Each patient received testolac- } \\
\text { tone } 500 \mathrm{mg} \text { orally four times per } \\
\text { day or placebo (gelatin capsules } \\
\text { containing cornstarch) for eight } \\
\text { months, followed by an alternate } \\
\text { therapy for an additional eight } \\
\text { months. }\end{array}$ & $\begin{array}{l}\text { Intervention group consisted of } \\
2.5 \mathrm{mg} \text { letrozole }(n=15) \text { or the } \\
\text { control }(n=14) \text { groups with } 1 \\
\text { mg anastrozole both taken orally } \\
\text { once daily }\end{array}$ & $\begin{array}{l}\text { Patients were randomly assigned } \\
\text { treatment to receive either letro- } \\
\text { zole } 2.5 \mathrm{mg} \text { once a day or placebo } \\
\text { (starch } 100 \mathrm{mg} \text { once a day) }\end{array}$ \\
\hline $\begin{array}{l}\text { Type of } \\
\text { outcomes }\end{array}$ & $\begin{array}{l}\text { Total estradiol and testosterone } \\
\text { levels during testolactone expo- } \\
\text { sure; sex hormone binding glob- } \\
\text { ulin (SHBG) capacity and free } \\
\text { E2; LH and; FSH serum concen- } \\
\text { trations }\end{array}$ & $\begin{array}{l}\text { Serum FSH, LH, prolactin (PRL), } \\
\mathrm{T} \text {, and E2 levels and; total motile } \\
\text { sperm count - ejaculate volume } x \\
\text { concentration } x \text { motile (fraction) }\end{array}$ & \\
\hline $\begin{array}{l}\text { Follow-up } \\
\text { (months) }\end{array}$ & 16 & 6 & 8 \\
\hline
\end{tabular}

\section{Included studies}

The three included studies (Clark \& Sherins, 1989; Gregoriou et al., 2012; Cavallini et al., 2013) comprised a total of 100 infertile men. Cavallini (2013) evaluated the largest number of patients $(n=46,46 \%)$ of the total sample, followed by Gregoriou et al. (2012) with $(n=29,29 \%)$ of the evaluated patients and, by Clark \& Sherins (1989) study with only $(n=25,25 \%)$ (Table 2$)$.

\section{Type of patients}

Clark \& Sherins (1989) assessed men that were partners in infertile marriages in which there was failure to conceive for at least two years prior to consideration of entry into the protocol. Each subject provided six or more semen samples during at least a 4-month period to confirm oligozoospermia. Only subjects with a mean sperm concentration of less than $20 \times 106$ per $\mathrm{mL}$ were accepted into the study. The median patients' ages were not reported.

Gregoriou et al. (2012) evaluated infertile men with low $\mathrm{T} / \mathrm{E}_{2}$ ratio $(<10)$. All patients had sperm concentrations $<10 \times 106$ spermatozoa/mL, and T levels < $300 \mathrm{ng} / \mathrm{dL}$. Testicular volume was measured with the use of ultrasound using the equation: length $X$ height $X$ width $X 0.71$ (Paltiel et al., 2002). The median patients' ages were not reported. Cavallini (2013) assessed non-smoker, non-obstructive azoospermic (NOA) patients who yielded no spermatozoa with fine needle aspiration and cryptozoospermic patients with T/E2 ratio < 10. Cavallini (2013) defined azoospermia as the absence of sperm in the pellets of two centrifuged semen samples collected 7-30 days apart and; cryptozoospermia was defined as the presence of sperm in the pellet (but not in the ejaculate) of at least one semen sample out of the two collected, i.e., with a sperm concentration $<10^{3} \mathrm{ml}$. The median age was 45 and 44 years old in the letrozole and placebo groups, respectively (Table 2).

There was no report about other comorbidities in the three studies included.

\section{Type of intervention and follow-up}

Cavallini (2013) randomly assigned the patients to received either letrozole $2.5 \mathrm{mg}$ once a day $(n=52)$ or placebo (starch $100 \mathrm{mg}$ once a day) for six months.

Gregoriou et al. (2012) allocated patients to the intervention group consisted of $2.5 \mathrm{mg}$ letrozole $(n=15)$ or the control $(n=14)$ groups with $1 \mathrm{mg}$ anastrozole, both taken orally once daily during also six months. 
Patients in the Clark \& Sherins study (1989) were followed for 16 months. Each patient received testolactone 500 $\mathrm{mg}$ orally four times per day or placebo (gelatin capsules containing cornstarch) for eight months, followed by an alternate therapy for an additional eight months (Table 2).

\section{Type of outcome measures}

Clark \& Sherins (1989) evaluated total estradiol and testosterone levels during testolactone exposure; sex hormone binding globulin (SHBG) capacity and free $\mathrm{T}$ levels; free E2; LH and FSH serum concentrations.

Gregoriou et al. (2012) assessed serum FSH, LH, prolactin (PRL), $T$, and $E_{2}$ levels and; total motile sperm counts - ejaculate volume $x$ concentration $x$ motile (fraction).

Cavallini (2013) measured sperm concentration and motility; the differences in FSH, LH, E2, T and PRL levels and; bilateral testicular volume (Table 2 ).

\section{Risk of bias in the included studies}

Gregoriou et al. (2012) used an alternating basis and, then we classified this domain as being under a high risk of bias. However, Clark \& Sherins (1989) and Cavallini (2013) studies presented a low risk of bias because they used a random number system and casual number tables, respectively.

With regards the allocation concealment both Clark \& Sherins (1989) and Gregoriou et al. (2012) studies were classified as low risk of bias because they used a third party (i.e., CRC Pharmacy and outpatient clinic, respectively) to keep the allocation safe. However, Cavallini (2013) study did not report if there was allocation concealment and, then classified it as unclear risk of bias.

In the study of Clark \& Sherins (1989) both investigator and patient were blinded to the sequence allocation and therefore we ranked it as low risk of bias; however, there was no description whether the outcome assessor were blinded to treatment allocation (unclear risk of bias). Gregoriou et al. (2012) was classified as unclear risk of bias for blinding of investigator, patient and outcome assessor because they did not report if there was any method to avoid detection bias. However, Cavallini (2013) used color-coded boxes to ensure blindness of both patients and investigators, and they also assigned each sample with a code number to ensure blinding of outcome assessor and, therefore we ranked this domain as low risk of bias.

Clark \& Sherins (1989) lost four and two patients in the letrozole and placebo groups, respectively; which was less than $20 \%$ and, therefore we classified it as low risk of bias. Gregoriou et al. (2012) study did not report whether there was drop-out and withdrawal, therefore we ranked it as an unclear risk of bias. On the other hand, Cavallini (2013) showed a low risk of bias since there was no incomplete outcome reports in the study (Figure 2 ).

\section{Effects of intervention}

It was not possible to perform meta-analysis as the included studies were not only heterogeneous with respect to intervention and outcomes but also there was insufficient data. Therefore, we decided to insert some available data into forest plots.

In the representation of meta-analysis with a single study (Clark \& Sherins., 1989) comparing testolactone at 16 months versus placebo, related to the hormone concentrations, there was a statistically significance difference favoring the use of testolactone for the following subcategories: LH (mIU/ml) (Mean Difference (MD) -3.50 [Confidential interval (CI) $95 \%-4.29$ to -2.71$]$ ); $\mathrm{FSH}$ (mIU/ $\mathrm{ml})(\mathrm{MD}-6.40$ [CI 95\% -7.29 to -5.51); E2 (mIU/ml) (MD -17.00 [CI 95\% -60.79 to 26.79]; free T $(\mathrm{mIU} / \mathrm{ml})$ (MD -4.40 [CI 95\% - 5.51 to -3.29], free E2 ( $\mathrm{mIU} / \mathrm{ml})$ (MD -0.09 [CI 95\% - 0.12 to -0.06$] ; 170 \mathrm{HP}(\mathrm{mIU} / \mathrm{ml})$ (MD)-
Figure 2. Risk of Bias

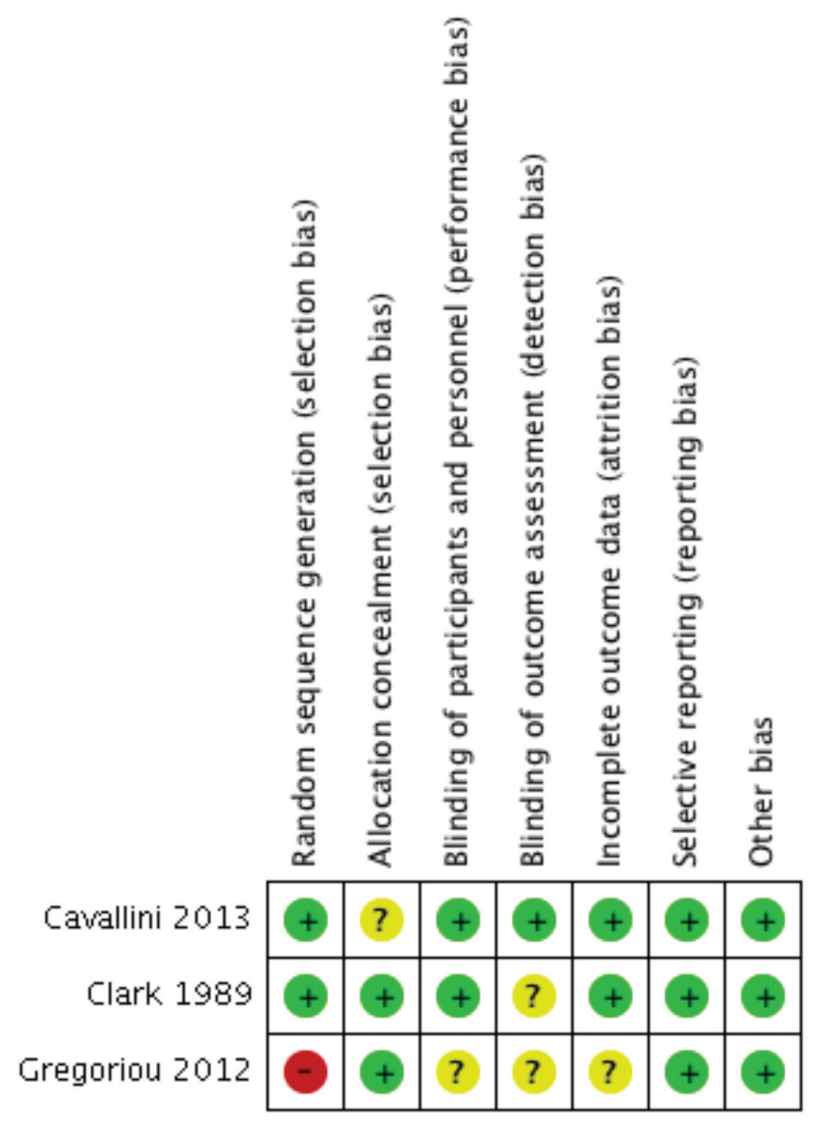

53.00 [CI 95\% -57.75 to -48.25 ]. However, for SHBG (ug/ dL) (MD 0.20 [CI 95\% 0.15 to 0.25 ] there was a statistically significance difference favoring placebo compared to testolactone, but there was no difference related to PRL (ng/ml) (MD 0.20 [CI 95\% -0.13 to 0.53] (Figure 3).

In the representation of meta-analysis from a single study (Gregoriou et al., 2012) comparing letrozole versus anastrozole at six months, there was a statistically significance difference favoring the use of anastrozole for serum LH (mIU/mL) (MD -1.73 [CI 95\% -2.94 to -0.52]. Howev$\mathrm{er}$, in the remaining subcategories, testicular volume $(\mathrm{mL})$; serum FSH (mIU/ $\mathrm{mL})$; serum $\mathrm{T}(\mathrm{ng} / \mathrm{dL})$; serum E2 (pg/ $\mathrm{mL}$ ); T/E2 ratio and; TFSF there were no statistically significance difference between the study groups (Figure 4).

In the representation of meta-analysis with a single study (Gregoriou et al., 2012) comparing letrozole versus anastrozole at six months, there was a statistically significance difference favoring the use of anastrozole compared to letrozole for increasing sperm count (X106) (MD -3.71 [CI 95\% -5.09 to -2.33] (Figure 5). However, in the remaining subcategories: TFSF ejaculate volume $(\mathrm{mL})$ and motility there were no statistically significance difference between the study groups.

\section{Adverse effects}

Clark \& Sherins (1989) reported that there were no significant changes in sperm density, motility or morphology, or in testicular size during the clinical trial (data not 
Figure 3. Representation of meta-analysis with a single study comparing testolactone versus placebo with regards hormone concentration in oligozoospermic infertile men at 16 months

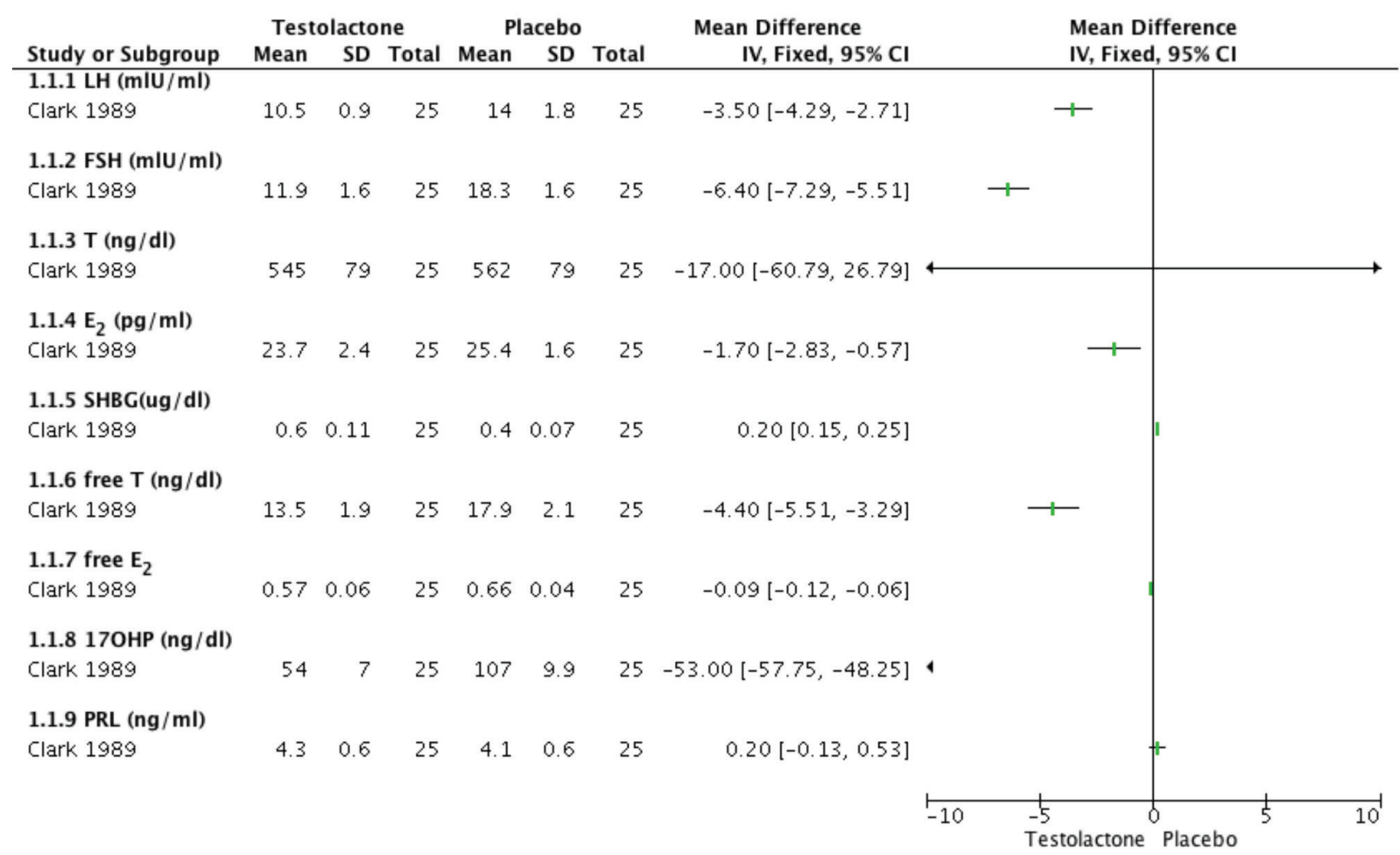

Figure 4. Representation of meta-analysis with a single study comparing letrozole versus anastrozole with regards hormonal concentration and testicular volume at six months

\begin{tabular}{|c|c|c|c|c|c|c|c|c|c|c|}
\hline \multirow{3}{*}{ Study or Subgroup } & \multicolumn{3}{|c|}{ Anastrazole } & \multicolumn{3}{|c|}{ Letrozole } & \multirow{3}{*}{$\begin{array}{l}\text { Mean Difference } \\
\text { IV, Fixed, } 95 \% \mathrm{CI}\end{array}$} & \multirow{2}{*}{\multicolumn{3}{|c|}{$\begin{array}{l}\text { Mean Difference } \\
\text { IV, Fixed, } 95 \% \mathrm{CI}\end{array}$}} \\
\hline & \multirow{2}{*}{$\frac{\text { Mean }}{\text { ne }(\mathrm{mL})}$} & \multirow[t]{2}{*}{ SD } & \multirow[t]{2}{*}{ Total } & \multirow[t]{2}{*}{ Mean } & \multirow[t]{2}{*}{ SD } & \multirow[t]{2}{*}{ Total } & & & & \\
\hline & & & & & & & & & & \\
\hline Gregoriou 2012 & 15.01 & 4.3 & 15 & 13.89 & 3.42 & 14 & $1.12[-1.70,3.94]$ & & & \\
\hline \multicolumn{11}{|c|}{ 2.1.2 Serum FSH $(\mathrm{mlU} / \mathrm{mL})$} \\
\hline Gregoriou 2012 & 8.41 & 1.95 & 15 & 8.45 & 1.93 & 14 & $-0.04[-1.45,1.37]$ & & & \\
\hline \multicolumn{11}{|c|}{ 2.1.3 Serum LH $(\mathrm{mlU} / \mathrm{mL})$} \\
\hline Gregoriou 2012 & 9.28 & 1.8 & 15 & 11.01 & 1.53 & 14 & $-1.73[-2.94,-0.52]$ & & & \\
\hline \multicolumn{11}{|c|}{ 2.1.4 Serum T $(\mathbf{n g} / \mathrm{dL})$} \\
\hline Gregoriou 2012 & 495 & 65 & 15 & 513 & 65 & 14 & $-18.00[-65.34,29.34]$ & $\longleftarrow$ & & \\
\hline \multicolumn{11}{|c|}{ 2.1.5 Serum $E_{2}(p g / m L)$} \\
\hline Gregoriou 2012 & 14.98 & 2.58 & 15 & 15.15 & 1.95 & 14 & $-0.17[-1.83,1.49]$ & & & \\
\hline \multicolumn{10}{|l|}{ 2.1.6 $\mathrm{T} / \mathrm{E}_{2}$ ratio } & \\
\hline \multicolumn{11}{|c|}{ 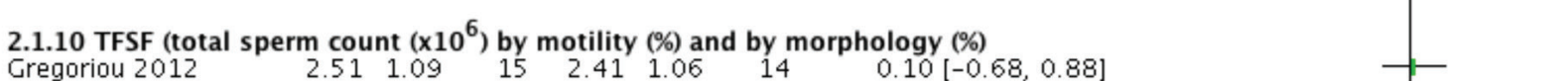 } \\
\hline & & & & & & & & $\begin{array}{ll}1 & -5 \\
-4 & -2 \\
\text { Anastrazole }\end{array}$ & Letrozole & 4 \\
\hline
\end{tabular}


Figure 5. Representation of meta-analysis with a single study comparing letrozole versus anastrozole with regards sperm quality at six months

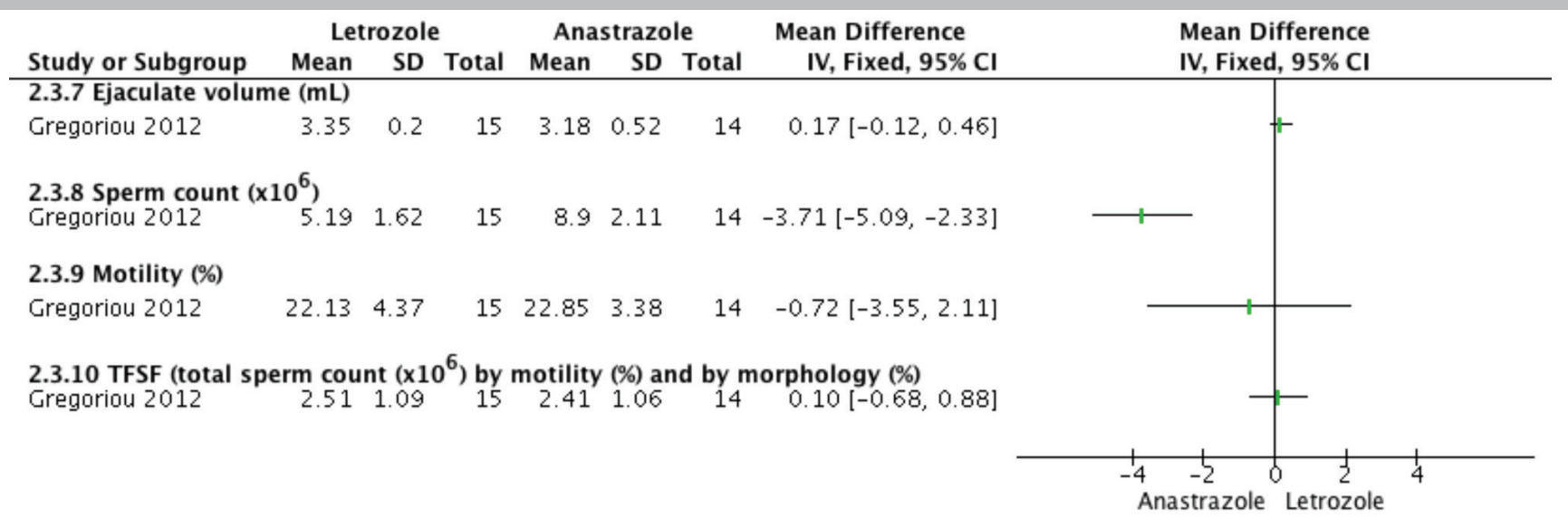

shown). Also, none of the women became pregnant during the study. However, three of the women became spontaneously pregnant 3,10 and 18 months after completion of the 16-month drug trial. No attempt was made to verify parentage in their pregnancies.

Gregoriou et al (2012) informed there was no improvement in seminal parameters in 4 of 15 patients in the letrozole group ( $26.6 \%)$ and in 3 of 14 patients in the anastrozole group ( $21.4 \%)$. Additionally, two patients complained of transient weakness, 1 patient of nausea that lasted for 10 days, and 2 patients of mild headache. One patient developed mild diarrhea at 1 month of use, which lasted for 3 days and subsided on its own without further sequelae; two patients developed transient nausea and one patient a mild headache. Cavallini (2013) reported that sperm concentration, sperm motility, FSH, LH and T significantly increased in Group 1 (letrozole) patients at 3 and 6 months (there was no significant difference between the 3- and the 6-month data), but no improvements were observed in Group 2 (placebo) patients. Conversely, E2 levels were significantly decreased in Group 1 patients at 3 and 6 months (there was no significant difference between the 3- and 6-month data), but no significant difference was demonstrated in Group 2 patients. No natural pregnancies occurred in either group. No significant modification in PRL levels occurred in either, Group 1 or Group 2 patients. The side effects were significantly higher in the group of patients treated with the active drug. Five patients demonstrated loss of libido and hair, two patients had cutaneous rashes and one patient reported only loss of libido. Spermatozoa could be found in the ejaculate of all NOA patients treated with letrozole, while the NOA patients treated with the placebo remained azoospermic.

\section{DISCUSSION}

Approximately $8 \%$ of men on reproductive age seek medical attention for infertility problems. Up to $10 \%$ of these men present with a reversible cause affecting their fertility potential. As such, the male partner must be systematically evaluated in every investigation of an infertile couple (Esteves et al., 2011). Testolactone acts on the inhibition of steroid aromatase activity and the reduction in estrone synthesis. Anastrozole and letrozole are members of a novel class of non-steroidal, hormone-targeting agents used for breast cancer therapy. They reversibly inhibit the aromatase enzyme, which converts the androgen precursors in adipose tissue to E2. Blocking estrogen production has been shown to provoke increased gonadotropin and androgen levels in the blood and a parallel E2 decrease, resulting in spermatogenesis stimulation (Raman \& Schlegel, 2002; Gregoriou et al., 2012).

This review offers up-to-date, but limited evidence about the effects of aromatase inhibitors in improving semen parameters in infertile men. The main features of this review are the comprehensive search strategy that allowed us to map the existing knowledge about this topic and the high-quality level of evidence considered in this review. However, there were only three studies included with a small sample size and different clinical outcomes evaluated.

Clark \& Sherins (1989) reported the use of testolactone to improve spermatogenesis. Despite the number of patients included in the study, there was a statistically significant difference favoring the use of the drug, compared to placebo, relating to hormones $\mathrm{LH}$; FSH; E2; free T; free $\mathrm{E} 2$ and $17 \mathrm{OHP}$ that play a crucial role on sperm production, specially FSH.

In another study Gregoriou et al. (2012) compared two different aromatase inhibitors, letrozole and anastrozole, and also noticed a statistically significant difference relating to sperm count and LH concentration favoring the use of anastrozole. LH acts on Leydig cells, stimulating the release of androgens which induce or maintain a male phenotype, stimulate sexual organs and androgen-controlled actions in the periphery. At the same time androgens function as feedback hormone at the hypothalamic level. In this scenario, if the use of anastrozole reduced the concentration of LH (Schlatt \& Ehmcke, 2014), it can be postulated that there is more free testosterone acting on the gonads and this may benefit spermatogenesis. The results indicate that although anastrozole and letrozole are both non-steroidal inhibitors, anastrozole proved to be more effective with respect to LH concentration and sperm count, with its dosage being relatively less than the dose of letrozole alone, and therefore should be the drug of choice for the treatment of infertile men when using aromatase inhibitors. Contradicting literature that affirms the effectiveness of letrozole compared to anastrozole to treat spermatogenesis impairment (Schlegel, 2012). Other randomized controlled trials of aromatase inhibitors would be beneficial to confirm these findings and better define the potential role of those agents in the treatment of male infertility.

\section{CONCLUSION}

The best available evidence, although based in very low quality, suggests that the use of testolactone is more effec- 
tive compared to placebo, related to the improvement of some of hormonal concentration in infertile men. Furthermore, the anastrozole presents more benefits compared to letrozole with regards to serum $\mathrm{LH}$ and sperm count. Further trials are needed with standardized interventions and outcomes to confirm these findings.

\section{Authors role}

MAR and MBR conceived the review. RED and WRS coordinated the review. MAR undertook manual searches, screened search results, screened retrieved papers against inclusion criteria, appraised quality of papers, abstracted data from papers, interpreted data and wrote the manuscript. LFOG organized retrieval of papers; wrote to authors of papers to ask for additional information; obtained and screened data on unpublished studies; managed data for the review and; wrote the manuscript. RED also screened retrieved papers against inclusion criteria; abstracted data from papers; interpreted data and; wrote the manuscript. WRS also obtained and screened data on unpublished studies; provided additional data about papers; and; wrote the manuscript. $\mathrm{CBJ}$ and $\mathrm{AK}$ wrote and approved the manuscript.

\section{CONFLICT OF INTERESTS}

No conflict of interest have been declared.

\section{Corresponding author:}

Mariana Antunes Ribeiro

Department of Morphology

Institute of Biosciences, Sao Paulo State University, UNESP

Botucatu/SP - Brazil

E-mail: ribeiro.mantunes@gmail.com

\section{REFERENCES}

Cavallini G, Biagiotti G, Bolzon E. Multivariate analysis to predict letrozole efficacy in improving sperm count of non-obstructive azoospermic and cryptozoospermic patients: a pilot study. Asian J Androl. 2013;15:806-11.

Clark R V, Sherins RJ. Treatment of men with idiopathic oligozoospermic infertility using the aromatase inhibitor, testolactone. Results of a double-blinded, randomized, placebo-controlled trial with crossover. J Androl. 1989; 10:240-7

Esteves SC, Miyaoka R, Agarwal A. An update on the clinical assessment of the infertile male. Clinics. 2011;66:691-700.

Gregoriou O, Bakas P, Grigoriadis C, Creatsa M, Hassiakos $D$, Creatsas G. Changes in hormonal profile and seminal parameters with use of aromatase inhibitors in management of infertile men with low testosterone to estradiol ratios. Fertil Steril. 2012;98:48-51.

Higgins JPT, Altman DG, Gøtzsche PC, Jüni P, Moher D, Oxman AD, Savovic J, Schulz KF, Weeks L, Sterne JAC. Cochrane Bias Methods Group; Cochrane Statistical Methods Group. The Cochrane Collaboration's tool for assessing risk of bias in randomised trials. BMJ. 2011;343:d5928.

Jarow JP, Zirkin BR. The androgen microenvironment of the human testis and hormonal control of spermatogenesis. Ann N Y Acad Sci. 2005;1061:208-20.

Moher D, Liberati A, Tetzlaff J, Altman DG; PRISMA Group. Preferred reporting items for systematic reviews and meta-analyses: the PRISMA statement. BMJ. 2009;339:b2535.

Paltiel HJ, Diamond DA, Canzio J Di, Zurakowski D, Borer JG, Atala A. Testicular volume: comparison of orchidometer and US measurements in dogs. Radiology. 2002; 222:114-9.

Pavlovich CP, King P, Goldstein M, Schlegel PN. Evidence of a treatable endocrinopathy in infertile men. J Urol. 2001; 165:837-41.

Raman JD, Schlegel PN. Aromatase inhibitors for male infertility. J Urol. 2002;167:624-9.

de Ronde W, de Jong FH. Aromatase inhibitors in men: effects and therapeutic options. Reprod Biol Endocrinol. 2011;9:93.

Santen RJ. Feedback control of luteinizing hormone and follicle-stimulating hormone secretion by testosterone and estradiol in men: physiological and clinical implications. Clin Biochem. 1981;14:243-51.

Schlatt S, Ehmcke J. Regulation of spermatogenesis: an evolutionary biologist's perspective. Semin Cell Dev Biol. 2014;29:2-16.

Schlegel PN. Aromatase inhibitors for male infertility. Fertil Steril. 2012;98:1359-62.

Stephens SM, Polotsky AJ. Big enough for an aromatase inhibitor? How adiposity affects male fertility. Semin Reprod Med. 2013;31:251-7.

Walker $\mathrm{WH}$, Cheng J. FSH and testosterone signaling in Sertoli cells. Reproduction. 2005;130:15-28. 